\title{
CÁC NHÂN TỐ ẢNH HỬ̛̉nG ĐẾN Ý ĐỊNH MUA THỰC PHẨM HŨ̃U CƠ CỦA NGƯỜI TIÊU DÙNG TẠI THÀNH PHỐ LONG XUYÊN
}

\author{
Huỳnh Đình Lệ Thu ${ }^{*}$, Nguyễn Thị Minh Thư ${ }^{2}$ và Hà Nam Khánh Giao ${ }^{3}$ \\ ${ }^{1}$ Trường Đại học An Giang, Đại học Quốc gia Thành phố Hồ Chí Minh \\ ${ }^{2}$ Sinh viên, Truờng Đại học An Giang, Đại học Quốc gia Thành phố Hồ Chí Minh \\ ${ }^{3}$ Học viện Hàng không Việt Nam \\ *Tác giả liên hệ: hdlthu@agu.edu.vn
}

\section{Lịch sử bài báo}

Ngày nhận: 30/6/2020; Ngày nhận chỉnh sưa: 26/8/2020; Ngày duyệt đăng: 19/11/2020

\section{Tóm tắt}

Nghiên cưu được thực hiện với muc đích xác định ảnh hưởng của thông tin minh bạch, kiến thức về thực phẩm hũu co đến thái độ, niềm tin của người tiêu dùng, tù đó cùng với chuẩn chủ quan ảnh hưởng đến $\dot{y}$ định mua thực phẩm hũu co. Dũ liệu được thu thập tù 238 nguoòi tiêu dùng tại thành phố Long xuyên có độ tuổi tù̀ 18 trở lên và có nghe nói đến thực phẩm hưu co. Phưong pháp phân tích EFA và cấu trúc tuyến tính SEM được dùng để đánh giá độ tin cậy của thang đo và kiểm định mô hình lý thuyết. Kết quả cho thấy, thái độ và niềm tin có mối quan hệ cùng chiều với ý định mua thực phẩm hũu co. Bên cạnh đó, nghiên cúu cho thấy niềm tin đóng vai trò nhu tiền đề của thái độ và làm trung gian trong mối quan hệ giũa thông tin minh bạch và kiến thức về thực phẩm hũu co với ý định mua thục phẩm hũu co:

Từ khóa: Kiến thức về thực phẩm hũu co; Long Xuyên, thực phẩm hũu co, thông tin minh bạch, Ý định mua.

\section{FACTORS INFLUENCING CONSUMERS' INTENTION OF BUYING ORGANIC FOOD IN LONG XUYEN CITY \\ Huynh Dinh Le Thu ${ }^{1 *}$, Nguyen Thi Minh Thu² and Ha Nam Khanh Giao ${ }^{3}$ \\ ${ }^{1}$ An Giang University, Viet Nam National University, Ho Chi Minh City \\ ${ }^{2}$ Student, An Giang University, Viet Nam National University, Ho Chi Minh City \\ ${ }^{3}$ Viet Nam Aviation Academy \\ *Corresponding author: hdlthu@agu.edu.vn}

\section{Article history}

Received: 30/6/2020; Received in revised form: 26/8/2020; Accepted: 19/11/2020

\section{Abstract}

This study was conducted to identify the effects of explicit information and perceived organic knowledge on their attitudes and trust, together with subjective norms influencing consumers' intention to buy organic foods. The data was collected from 238 consumers in Long Xuyen city, who were 18 years of age and older, having heard of organic food. Exploratory Factor Analysis (EFA) and Structural Equation Modeling (SEM) medthods were used to validate the instrumental scale and theoretical testing models. The results showed that attitudes and trust have a positive relationship with intentions of organic-food purchase. Besides, trust, serving as the antecedent of attitudes, significantly mediates the relationships between explicit information, perceived organic knowledge, and organic purchase intentions.

Keywords: Explicit information, Long Xuyen city, organic food, perceived organic knowledge, purchase intentions. 


\section{1. Đặt vấn đề}

Các tác nhân gây ảnh hưởng đến sức khỏe người tiêu dùng và môi trường từ việc sử dụng thuốc trừ sâu, các sinh vật biến đổi gen và các chất phi tự nhiên khác trong sản xuất nông nghiệp nhằm gia tăng sản lượng đã và đang thúc đẩy người tiêu dùng và các nhà tiếp thị quan tâm nhiều hơn đến thực phẩm hữu cơ (Teng và Wang, 2015). Riêng ở nước ta, theo thống kê được đăng tải trên báo Thời Nay, năm 2019 đã xảy ra 58 vụ ngộ độc thực phẩm với 1668 người mắc và 9 người tử vong vì thế nhu cầu tiêu dùng các thực phẩm an toàn đảm bảo cho sức khỏe ngày càng trở nên quan trọng hơn bao giờ hết. Người tiêu dùng ngày càng quan tâm đến việc lựa chọn thực phẩm tiêu dùng hàng ngày (Thu Lưu, 2019). Trên thực tế, tiêu thụ thực phẩm hữu cơ đã trở thành một xu hướng mới ở Việt Nam. Thậm chí, trong vài năm trở lại đây, các sản phẩm hữu cơ đang tạo nên cơn sốt đối với một bộ phận người tiêu dùng. Rau, cá, thịt, hoa quả hữu cơ đều nằm trong danh sách thực phẩm được các bà nội trợ lựa chọn cho bữa ăn gia đình.

Khi xem xét các lý thuyết liên quan đến tiêu thụ thực phẩm hữu cơ, nhiều nghiên cứu đã xem xét động cơ mua thực phẩm hữu cơ chẳng hạn Agarwal (2019), Secapramana và Katargo (2019), Effendi và cs. (2015), Teng và Wang (2015), Švecová và Odehnalová (2019), Hughner và cs. (2007). Nghiên cứu của Hughner và cs. (2007) kết luận rằng ý định mua thực phẩm hữu cơ bao gồm các mối quan tâm về sức khỏe, môi trường, an toàn thực phẩm và sự bảo vệ động vật, những mong muốn hỗ trợ nền kinh tế địa phương và một cuộc sống khỏe mạnh. Tuy nhiên, các nghiên cứu trước không tập trung nhiều vào các yếu tố có thể giúp người tiêu dùng tạo ra niềm tin và thái độ tích cực đối với thực phẩm hữu cơ, hoặc khám phá sự kết hợp giữa các yếu tố có thể làm tăng mức tiêu dùng thực phẩm hữu cơ (Teng và Wang, 2015). Các tác giả đã lập luận rằng niềm tin là một điều kiện tiên quyết giúp một doanh nghiệp thành công vì người tiêu dùng thường do dự khi thực hiện hành vi mua, trừ khi họ tin tưởng vào người bán (Kim và cs. 2008). Niềm tin của người tiêu dùng có thể còn quan trọng hơn trong quyết định mua thực phẩm hữu cơ so với thực phẩm thông thường. Niềm tin là điều cần thiết đối với hành vi mua thực phẩm hữu cơ (Teng và Wang, 2015).

Ở Việt Nam, các nghiên cứu về ý định mua thực phẩm hữu cơ cũng đã được thực hiện ở những bối cảnh khác nhau và tìm thấy các yếu tố ảnh hưởng đến ý định mua như thái độ, chuẩn chủ quan, niềm tin, sự quan tâm sức khỏe, mức độ quan tâm môi trường, nhận thức về chất lượng, khả năng kiểm soát hành vi,...(Nguyễn Kim Nam, 2015; Hoàng Thị Bảo Thoa và cs., 2019; Trịnh Thùy Anh, 2014). Tuy nhiên, số lượng các nghiên cứu còn hạn chế và chưa đưa ra được kết luận chung về các yếu tố ảnh hưởng đến ý định mua thực phẩm hữu cơ của người tiêu dùng tại Việt Nam cũng như tại các địa phương. Trong những năm trở lại đây nhu cầu tiêu dùng thực phẩm an toàn tại thành phố Long Xuyên ngày càng gia tăng, mỗi ngày thành phố cung cấp ra thị trường khoảng 2,5 tấn rau an toàn (Ánh Nguyên, 2018). Điều này cho thấy xu hướng tiêu dùng các thực phẩm an toàn được người tiêu dùng ngày càng quan tâm nhiều hơn trong quyết định tiêu dùng thực phẩm của họ. Người tiêu dùng tại thành phố Long Xuyên ngày càng quan tâm đến nguồn gốc và sự an toàn từ các thực phẩm họ tiêu dùng. Vì vậy, nghiên cứu này nhằm tìm hiểu các nhân tố quan trọng tác động đến ý định mua thực phẩm hữu cơ của người tiêu dùng tại thành phố Long Xuyên để cung cấp ý nghĩa và làm cơ sở để các nhà kinh doanh trong lĩnh vực thực phẩm hữu cơ có các chiến lược hiệu quả để phát triển thị trường thực phẩm hữu cơ tại thành phố Long Xuyên.

\section{Tổng quan lý thuyết, lược sử nghiên cứu và phát triển giả thuyết}

\subsection{Tổng quan lý thuyết, lược sử tài liệu}

Các nhà nghiên cứu đã sử dụng rộng rãi lý thuyết hành vi hợp lý (Ajzen và Fishbein, 1980; Fishbein và $A j z e n, 1975)$ và lý thuyết hành vi có kế hoạch (Ajzen, 1989, 1991) để giải thích ý 
định mua thực phẩm hữu cơ của người tiêu dùng (Agarwal, 2019; Teng và Wang, 2014; Effendi và cs., 2015; Secapramana và Katargo, 2019; Kim và Chung, 2011; Hoàng Thị Bảo Thoa và cs., 2019).

Lý thuyết hành vi hợp lý được xây dựng bởi Fishbein và Ajzen (1975). Theo lý thuyết hành vi hợp lý, một trong những yếu tố quan trọng nhất để xác định hành vi của con người là ý định thực hiện hành vi. Mối quan hệ giữa ý định hành vi và hành vi của con người được sử dụng đồng thời để nghiên cứu hành vi mua của người tiêu dùng. Lý thuyết hành vi hợp lý chỉ ra rằng ý định hành vi của một người chịu ảnh hưởng bởi hai thành phần chính: Thái độ cá nhân và chuẩn chủ quan.

Tương tự như vậy, Ajzen (1991) đã xây dựng lý thuyết hành vi có kế hoạch, lý thuyết là phần mở rộng của lý thuyết hành vi hợp lý (Fishbein và Ajzen, 1975). Theo lý thuyết hành vi có kế hoạch, ba yếu tố cơ bản dùng để dự đoán ý định mua của người tiêu dùng là (1) Chuẩn chủ quan (được hiểu là tầm quan trọng của những ý kiến từ những người xung quanh mà người tiêu dùng quan tâm); (2) Thái độ của người tiêu dùng; (3) Nhận thức kiểm soát hành vi (tức là người tiêu dùng nhận thấy rằng họ có thể kiểm soát hành vi của mình) (Ajzen, 1991).

Bảng 1. Tổng hợp một số nghiên cứu trên thế giới và trong nước có liên quan

\begin{tabular}{lllcl}
\hline STT Đề tài nghiên cứu Năm $\quad$ Tác giả & $\begin{array}{c}\text { Quốc gia/ } \\
\text { Thành phố }\end{array}$ & Thang đo/Khám phá mới \\
\hline
\end{tabular}

\section{NGHIÊN CỨU TRÊN THẾ GIỚI}

\begin{tabular}{|c|c|c|c|c|c|}
\hline 1 & $\begin{array}{l}\text { Các nhân tố tác động đến } \\
\text { ý định mua thực phẩm hữu } \\
\text { cơ của người tiêu dùng tại } \\
\text { Đài Loan }\end{array}$ & 2015 & Teng và Wang & Đài Loan & $\begin{array}{l}\text { Thang đo: } \\
\text { + Chuẩn chủ quan } \\
\text { + Thái độ } \\
\text { + Niềm tin } \\
\text { + Thông tin minh bạch } \\
\text { + Kiến thức về thực phẩm } \\
\text { hữu cơ } \\
\text { + Ý định mua }\end{array}$ \\
\hline 2 & $\begin{array}{l}\text { Các nhân tố ảnh hưởng đến } \\
\text { ý định mua thực phẩm hữu } \\
\text { cơ của người tiêu dùng ở } \\
\text { Indonesia }\end{array}$ & 2019 & $\begin{array}{c}\text { Secapramana } \\
\text { và Katargo }\end{array}$ & Indonesia & $\begin{array}{l}\text { Thang đo: } \\
\text { + Chuẩn chủ quan } \\
\text { + Thái độ } \\
\text { + Niềm tin } \\
\text { + Thông tin minh bạch } \\
\text { +Kiến thức vềthực phẩm hữucơ } \\
\text { + Ý định mua }\end{array}$ \\
\hline 3 & $\begin{array}{l}\text { Các yếu tố quyết định hành } \\
\text { vi tiêu dùng của sinh viên } \\
\text { đến từ Brno khi mua thực } \\
\text { phẩm hữu cơ }\end{array}$ & 2019 & $\begin{array}{l}\text { Švecová và } \\
\text { Odehnalová }\end{array}$ & Brno & $\begin{array}{l}\text { Các yếu tố tác động: } \\
\text { + Thái độ cá nhân } \\
\text { + Chuẩn chủ quan } \\
\text { + Các khía cạnh đạo đức } \\
\text { + Sự quan tâm sức khỏe }\end{array}$ \\
\hline 4 & $\begin{array}{l}\text { Lý thuyết hành vi hợp lý và } \\
\text { ý định mua thực phẩm hữu } \\
\text { cơ ở Ấn Độ }\end{array}$ & 2019 & Agarwal & Ấn Độ & $\begin{array}{l}\text { Các yếu tố tác động: } \\
\text { + Thái độ } \\
\text { + Chuẩn chủ quan }\end{array}$ \\
\hline
\end{tabular}




\begin{tabular}{|c|c|c|c|c|c|}
\hline 5 & $\begin{array}{l}\text { Phân tích hành vi tiêu dùng } \\
\text { thực phẩm hữu cơ của người } \\
\text { tiêu dùng tại Bắc thị trấn } \\
\text { Sumatra, Indonesia }\end{array}$ & 2015 & Effendi và $c s$. & Indonesia & $\begin{array}{l}\text { Thái độ chịu ảnh hưởng của } \\
\text { kiến thức sản phẩm và kiến } \\
\text { thức sức khỏe }\end{array}$ \\
\hline \multicolumn{6}{|c|}{ NGHIÊN CÚU TẠI VIỆT NAM } \\
\hline 6 & $\begin{array}{l}\text { Các nhân tố ảnh hưởng đến } \\
\text { ý định mua thực phẩm hữu } \\
\text { cơ của người tiêu dùng ở } \\
\text { Hà Nội }\end{array}$ & 2019 & $\begin{array}{l}\text { Hoàng Thị } \\
\text { Bảo Thoa } \\
\text { và cs. }\end{array}$ & Hà Nội & $\begin{array}{l}\text { Thông tin rõ ràng trên nhãn } \\
\text { thực phẩm hữu cơ có tác động } \\
\text { cùng chiều đến thái độ và niềm } \\
\text { tin về thực phẩm hữu cơ }\end{array}$ \\
\hline 7 & $\begin{array}{l}\text { Ý định tiêu dùng thực phẩm } \\
\text { hữu cơ của người tiêu dùng } \\
\text { trẻ: Vai trò của niềm tin }\end{array}$ & 2015 & $\begin{array}{c}\text { Nguyễn Kim } \\
\text { Nam }\end{array}$ & Hồ Chí Minh & $\begin{array}{l}\text { Đối với nhóm người có niềm } \\
\text { tin cao thì ý định mua cao hơn } \\
\text { nhóm người có niềm tin thấp }\end{array}$ \\
\hline 8 & $\begin{array}{l}\text { Các yếu tố ảnh hưởng đến ý } \\
\text { định mua thực phẩm hữu cơ } \\
\text { của người tiêu dùng tại thành } \\
\text { phố Hồ Chí Minh }\end{array}$ & 2015 & $\begin{array}{l}\text { Hồ Thị Diệp } \\
\text { Quỳnh Châu }\end{array}$ & Hồ Chí Minh & $\begin{array}{l}\text { Các yếu tố tác động: } \\
\text { + Sự quan tâm đến môi } \\
\text { trường } \\
\text { + Sự quan tâm đến sức khỏe } \\
\text { + Kiến thức về thực phẩmhữu cơ } \\
\text { + Nhận thức về chất lượng } \\
\text { + Chuẩn chủ quan } \\
\text { + Nhận thức sự sẵn có } \\
\text { + Nhận thức giá }\end{array}$ \\
\hline 9 & $\begin{array}{l}\text { Các nhân tố tác động đến ý } \\
\text { định mua thực phâmm hữu cơ } \\
\text { của người tiều dùng tại thành } \\
\text { phố Đà Nẵng }\end{array}$ & 2017 & $\begin{array}{c}\text { Lê Thị Thùy } \\
\text { Dung }\end{array}$ & Đà Nẵng & $\begin{array}{l}\text { Các nhân tố tác động: } \\
\text { + Thái độ } \\
\text { + Sự quan tâm sức khỏe } \\
\text { + Sự quan tâm môi trường } \\
\text { + Niềm tin } \\
\text { + Sự sẵn có } \\
\text { + Giá } \\
\text { + Truyền thông đại chúng }\end{array}$ \\
\hline 10 & $\begin{array}{l}\text { Các yếu tố ảnh hưởng đến } \\
\text { quyết định mua thực phầm } \\
\text { hữu cơ của khách hàng trên } \\
\text { địa bàn thành phố Hồ Chí } \\
\text { Minh }\end{array}$ & 2014 & $\begin{array}{c}\text { Trịnh Thùy } \\
\text { Anh }\end{array}$ & Hồ Chí Minh & $\begin{array}{l}\text { Các yếu tố tác động: } \\
\text { + Ý thức về sức khỏe } \\
\text { +Kiến thức về thựcphẩm hữu cơ } \\
\text { + Giá cả } \\
\text { + Giá trị thông tin }\end{array}$ \\
\hline
\end{tabular}

Nguồn: Nhóm tác giả tổng hợp.

\subsection{Phát triển giả thuyết}

\section{- Thái độ}

Teng và Wang (2015) cho rằng thái độ tích cực đối với thực phẩm hữu cơ là một tiền đề quan trọng thúc đẩy ý định mua thực phẩm hữu cơ. Theo lý thuyết hành vi có kế hoạch, thái độ được xem như một yếu tố quan trọng quyết định đến ý định hành vi và thái độ đối với hành vi của một cá nhân càng tích cực thì ý định thực hiện hành vi của cá nhân đó càng mạnh (Ajzen, 1991). Từ các cơ sở trên, nghiên cứu đề xuất giả thuyết như sau:

Giả thuyết $H_{I}$. Thái độ có mối quan hệ cùng chiều với ý định mua thực phẩm hữu co.

\section{- Chuẩn chủ quan}


Theo Teng và Wang (2015), chuẩn chủ quan là những áp lực xã hội tác động lên nhận thức của cá nhân trong việc thực hiện hay không nên thực hiện hành vi. Theo Ajzen (2002), chuẩn chủ quan được xác định bởi niềm tin về việc những người có ảnh hưởng cho rằng cá nhân này nên thực hiện hành vi (nhóm người đó có thể là gia đình, bạn bè và những người có ảnh hưởng khác). Ajzen và Fishbein (1980) khẳng định chuẩn chủ quan là một yếu tố quan trọng quyết định sự ảnh hưởng của xã hội đối với ý định hành vi. Từ các cơ sở trên, nghiên cứu đề xuất giả thuyết như sau:

Giả thuyêt $H_{2}$. Chuẩn chủ quan có mối quan hệ cùng chiều với ý định mua thưc phẩm hũu co.

- Niềm tin

Niềm tin được xem như một trạng thái dễ bị tổn thương hoặc rủi ro nhận thấy xuất phát từ sự không chắc chắn của cá nhân liên quan đến động cơ, ý định và hành động tiềm năng của những người có ảnh hưởng mà người tiêu dùng phụ thuộc vào họ (Kramer, 1999). Trong nhiều trường hợp, niềm tin được dựa trên kinh nghiệm trước đây, niềm tin được xem như một cơ chế chung để giảm sự rủi ro trong nhận thức khi thực hiện các hành động bằng cách tăng kỳ vọng về một kết quả tích cực và sự chắc chắn trong nhận thức về ý định hành vi (Gefen, 2004).

Hart và Saunders (1997) chỉ ra rằng niềm tin là một trong những cách thức hiệu quả nhất để giảm sự không chắc chắn của người tiêu dùng. Do đó, tầm quan trọng của niềm tin trong tiêu dùng thực phẩm hữu cơ, niềm tin vào thực phẩm hữu cơ, cũng như các nhà cung cấp và chứng chỉ của họ có ảnh hưởng lớn đến thái độ của người tiêu dùng và ý định hành vi (Teng và Wang, 2015).

Giả thuyết $H_{3}$. Niềm tin có mối quan hệcùng chiều với thái độ về thục phẩm hũu co:

Giả thuyết $H_{4}$. Niềm tin có mối quan hệ cùng chiều với ý định mua thực phẩm hũu co.

- Thông tin minh bạch

Theo Teng và Wang (2015), việc người tiêu dùng tiếp cận được các thông tin minh bạch và đáng tin cậy là rất quan trọng đối với quá trình quyết định mua hàng. Những lợi ích và thông tin có liên quan về các sản phẩm thực phẩm hữu cơ cần được cung cấp đầy đủ cho người tiêu dùng, để giúp họ đưa ra quyết định hợp lý dựa trên ngân sách và/hoặc sở thích (Vermeir và Verbeke, 2006). Nghiên cứu của Gracia và Magistris (2008), Howard, Shay, và Green (1988) cho rằng việc cung cấp thông tin đầy đủ trên các sản phẩm thực phẩm hữu cơ là rất quan trọng để gia tăng nhu cầu tiêu dùng thực phẩm hữu cơ vì những thông tin này có thể làm tăng niềm tin của người tiêu dùng và thái độ đối với sản phẩm hữu cơ.

Giả thuyết $H_{5}$. Thông tin minh bạch trên nhãn thưc phẩm hũu cơ có mối quan hệ cùng chiều với niềm tin vào thực phẩm hưu co.

Giả thuyết $H_{6}$. Thông tin minh bạch trên nhãn thực phẩm hũu co có mối quan hệ cùng chiều với thái độ về thực phẩm hũu co.

- Kiến thức về thực phẩm hữu cơ

Theo Trịnh Thùy Anh (2014), kiến thức thực phẩm hữu cơ dùng để chỉ mức độ hiểu biết của người tiêu dùng về cấu trúc và bản chất của thực phẩm hữu cơ. Gracia và Magistris (2008) đã phát hiện ra rằng kiến thức hữu cơ không chỉ có thể làm tăng thái độ và khả năng mua thực phẩm hữu cơ mà còn có thể nâng cao mức chi tiêu của người tiêu dùng hiện có.

Bên cạnh đó, kiến thức nhận thức về thực phẩm hữu cơ được công nhận là một tiền đề quan trọng của niềm tin người tiêu dùng đối với thực phẩm hữu cơ (O'Fallon và cs., 2007). Demeritt (2002) cũng chỉ ra rằng không đủ kiến thức và nhận thức về thực phẩm hữu cơ được coi là rào cản quan trọng đối với quyết định mua thực phẩm hữu cơ.

Giả thuyết $H_{7}$ Kiến thức về thực phẩm hưu cơ có mối quan hệ cùng chiều với thái độ về thực phẩm hũu co:

Giả thuyết $H_{8}$. Kiến thưc về thực phẩm hũu cơ có mối quan hệ cùng chiều với niềm tin đối vào thực phẩm hữu co. 
3. Mô hình nghiên cứu và phương pháp nghiên cứu

\subsection{Mô hình nghiên cứu}

Nghiên cứu này kế thừa mô hình ý định mua thực phẩm hữu cơ được thực hiện bởi Teng và Wang (2015) gồm các yếu tố (1) Chuẩn chủ quan, (2) Thái độ, (3) Niềm tin, (4) Thông tin minh bạch, (5) Kiến thức về thực phẩm hữu cơ, (6) Ý định mua. Nghiên cứu cũng kết hợp các lý thuyết liên quan đến ý định mua và các nghiên cứu trước đây về ý định mua thực phẩm hữu co như là cơ sở lý thuyết để thực hiện nghiên cứu.

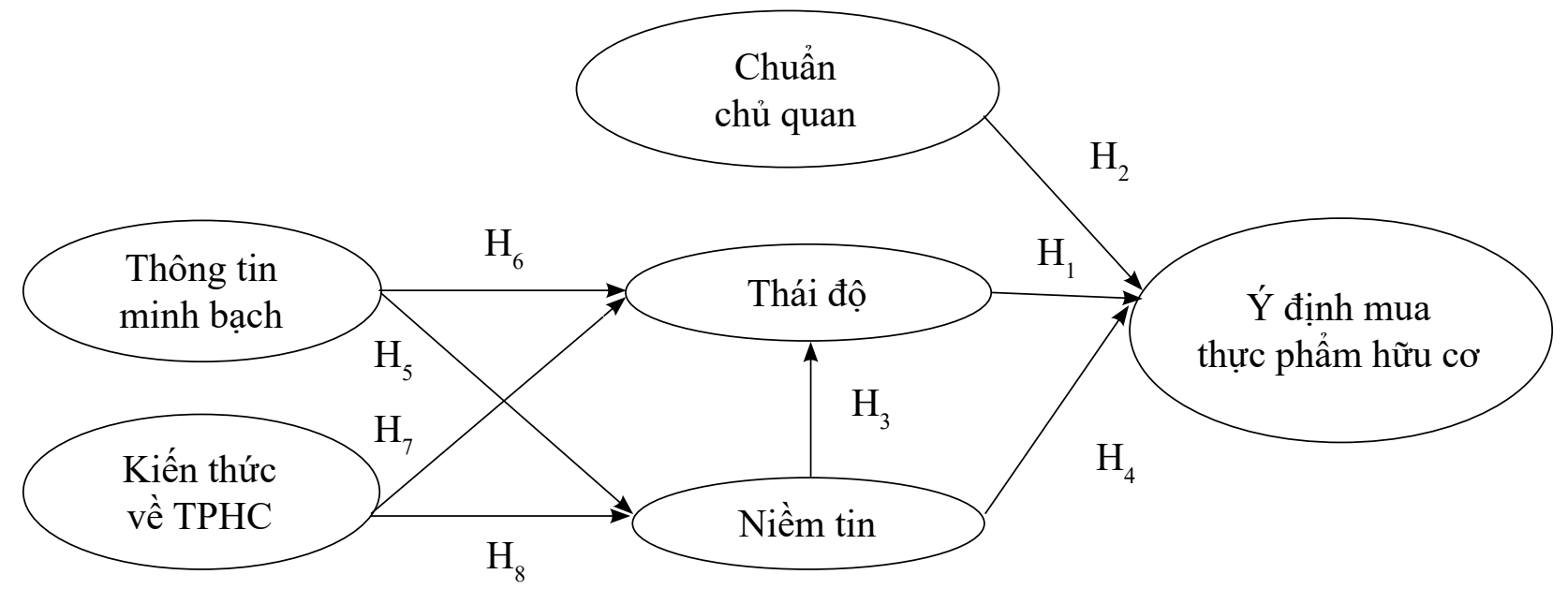

Hình 1. Mô hình nghiên cứu đề xuất

Nguồn: Teng và Wang (2015).

\subsection{Phương pháp nghiên cứu}

Nghiên cứu được thực hiện thông qua 2 giai đoạn: (1) Nghiên cứu định tính được thực hiện với kỹ thuật thảo luận tay đôi với 16 người tiêu dùng có kiến thức về thực phẩm hữu cơ tại thành phố Long Xuyên, (2) Nghiên cứu định lượng được thực hiện thông qua bảng câu hỏi chi tiết, với kỹ thuật lấy mẫu thuận tiện với 238 (n=238) người tiêu dùng tại thành phố Long Xuyên có độ tuổi từ 18 trở lên, và có nghe nói đến thực phẩm hữu cơ. Phương pháp phân tích EFA, phân tích nhân tố CFA và cấu trúc tuyến tính SEM được dùng để đánh giá độ tin cậy của thang đo và kiểm định mô hình lý thuyết. Thang đo trong nghiên cứu này kế thừa từ các nghiên cứu trước, có điều chỉnh và bổ sung cho phù hợp với thực tế nghiên cứu. Các thang đo sử dụng dạng Likert 5 mức độ tương ứng với mức từ "hoàn toàn không đồng ý" $=1$ đến "hoàn toàn đồng ý" $=5$.

Bảng 2. Diễn giải các biến quan sát trong mô hình nghiên cứu

\begin{tabular}{|c|c|c|}
\hline $\begin{array}{l}\text { Khái } \\
\text { niệm }\end{array}$ & Tên biến quan sát & Nguồn \\
\hline \multirow{4}{*}{ Thái độ } & $\begin{array}{l}\text { Thực phẩm hữu cơ có ít dư lượng hóa học hơn thực phẩm thông } \\
\mathrm{TD}_{1} \text { thường. }\end{array}$ & \multirow{4}{*}{$\begin{array}{c}\text { Gil và cs. }(2000) \\
\text { Lockie } v a ̀ \text { cs } \\
(2004)\end{array}$} \\
\hline & $\mathrm{TD}_{2}$ Tiêu dùng thực phẩm hữu cơ an toàn hơn thực phẩm thông thường. & \\
\hline & $\begin{array}{l}\text { Tiêu dùng thực phẩm hữu cơ tốt cho sức khỏe hơn thực phẩm thông } \\
\mathrm{TD}_{3} \text { thường. }\end{array}$ & \\
\hline & $\mathrm{TD}_{4}$ Thực phẩm hữu cơ có vị ngon hơn thực phẩm thông thường. & \\
\hline
\end{tabular}


TD 5 Thực phẩm hữu cơ có chất lượng cao hơn thực phẩm thông thường.

$\mathrm{TD}_{6}$ Thực phẩm hữu cơ đắt tiền hơn thực phẩm thông thường.

$\mathrm{TD}_{7}$ Thực phẩm hữu cơ nhìn hấp dẫn hơn thực phẩm thông thường.

$\mathrm{CQ}_{1}$ Gia đình tôi nghĩ rằng tôi nên mua thực phẩm hữu cơ.

$\mathrm{CQ}_{2}$ Bạn bè, đồng nghiệp của tôi nghĩ rằng tôi nên mua thực phẩm hữu cơ.

Chuẩn Tin tức, tạp chí và quảng cáo nói về thực phẩm hữu cơ ảnh hưởng

chủ quan $\mathrm{CQ}_{3}$ đến quyết định mua thực phẩm hữu cơ của tôi.

Chen (1998)

$\mathrm{CQ}_{4}$ Hỗ trợ của chính phủ cho thực phẩm hữu cơ ảnh hưởng đến quyết định mua thực phẩm hữu cơ của tôi.

\begin{tabular}{|c|c|c|c|}
\hline \multirow{4}{*}{ Niềm tin } & $\mathrm{NT}_{1}$ & $\begin{array}{l}\text { Tôi nghĩ rằng các công ty trong lĩnh vực thực phẩm hữu cơ nhận } \\
\text { thức được trách nhiệm của họ. }\end{array}$ & \multirow{4}{*}{$\begin{array}{c}\text { Krystallis và } \\
\text { Chryssohoidis } \\
\text { (2005), Siegrist } \\
\text { (2000) }\end{array}$} \\
\hline & $\mathrm{NT}_{2}$ & $\begin{array}{l}\text { Tôi tin tưởng những người bán thực phẩm hữu cơ có chứng nhận } \\
\text { chất lượng. }\end{array}$ & \\
\hline & $\mathrm{NT}_{3}$ & Tôi tin tưởng chất lượng thực phẩm hữu cơ có bao bì hoặc logo. & \\
\hline & $\mathrm{NT}_{4}$ & Tôi tin tưởng các tổ chức chứng nhận thực phẩm hữu cơ. & \\
\hline \multirow{4}{*}{$\begin{array}{l}\text { Thông tin } \\
\text { minh bạch }\end{array}$} & $\mathrm{TT}_{1}$ & $\begin{array}{l}\text { Nhãn mác, bao bì của thực phẩm hữu cơ cung cấp thông tin chính } \\
\text { xác về sản phẩm. }\end{array}$ & \multirow{4}{*}{$\begin{array}{l}\text { Doll và } \\
\text { Torkzadeh }\end{array}$} \\
\hline & $\mathrm{TT}_{2}$ & $\begin{array}{l}\text { Nhãn mác, bao bì của thực phẩm hữu cơ cung cấp đúng thông tin về } \\
\text { ngày sản xuất sản phẩm. }\end{array}$ & \\
\hline & $\mathrm{TT}_{3}$ & $\begin{array}{l}\text { Nhãn mác, bao bì của thực phẩm hữu cơ cung cấp đầy đủ thông tin } \\
\text { về sản phẩm. }\end{array}$ & \\
\hline & $\mathrm{TT}_{4}$ & $\begin{array}{l}\text { Tôi hài lòng với những thông tin trên nhãn mác, bao bì của thực } \\
\text { phẩm hữu cơ cung cấp. }\end{array}$ & \\
\hline \multirow{7}{*}{$\begin{array}{l}\text { Kiến thức } \\
\text { về thực } \\
\text { phẩm } \\
\text { hữu cơ }\end{array}$} & $\mathrm{KT}_{1}$ & Cá nhân tôi rất am hiểu về thực phẩm hữu cơ. & \multirow{7}{*}{$\begin{array}{l}\text { Chen (2008); } \\
\text { Effendi và cs. } \\
\quad \quad(2015)\end{array}$} \\
\hline & $\mathrm{KT}_{2}$ & $\begin{array}{l}\text { Trung bình người ở thành phố Long Xuyên là rất am hiểu về thực } \\
\text { phẩm hữu cơ. }\end{array}$ & \\
\hline & $\mathrm{KT}_{3}$ & Chính phủ rất am hiểu về thực phẩm hữu cơ. & \\
\hline & $\mathrm{KT}_{4}$ & Ngành công nghiệp thực phẩm rất am hiểu về thực phẩm hữu cơ. & \\
\hline & $\mathrm{KT}_{5}$ & Tôi biết quá trình sản xuất ra thực phẩm hữu cơ. & \\
\hline & $\mathrm{KT}_{6}$ & Tôi có thể phân biệt thực phẩm hữu cơ so với thực phẩm thông thường. & \\
\hline & $\mathrm{KT}_{7}$ & Tôi có thể nhận diện được bao bì, nhãn mác thực phẩm hữu cơ. & \\
\hline \multirow{3}{*}{$\begin{array}{l}\text { Ý định } \\
\text { mua }\end{array}$} & $\mathrm{YD}_{1}$ & $\begin{array}{l}\text { Nếu thực phẩm hữu cơ có sã̃n tại các siêu thị, cửa hàng thực phẩm } \\
\text { tôi sẽ mua chúng. }\end{array}$ & \multirow{3}{*}{$\begin{array}{l}\text { Schifferstein và } \\
\text { Oude Ophuis } \\
\text { (1998), Bredahl } \\
\text { (2001) }\end{array}$} \\
\hline & $\mathrm{YD}_{2}$ & $\begin{array}{l}\text { Tôi sẵn sàng mua thực phẩm hữu cơ mặc dù giá cao hơn thực phẩm } \\
\text { thông thường. }\end{array}$ & \\
\hline & $\mathrm{YD}_{3}$ & Xác suất tôi sẽ mua thực phẩm hữu cơ là rất cao & \\
\hline
\end{tabular}




\section{Kết quả nghiên cứu}

\section{1. Đánh giá độ tin cậy của thang đo}

Kết quả kiểm định Cronbach's Alpha đối với các thang đo cho thấy tất cả các thang đo đều đạt yêu cầu về độ tin cậy $(\geq 0,6)$. Các hệ số tương quan biến - tổng (Corrected Item - Total Correlation) đều cao hơn mức cho phép $(\geq 0,3)$ (Hà Nam Khánh Giao và Bùi Nhất Vương, 2019).

Bảng 3. Kết quả Cronbach's Alpha các thang đo

\begin{tabular}{|c|l|c|c|c|c|}
\hline \multicolumn{1}{|c|}{ STT } & \multicolumn{1}{|c|}{ Khân tố } & Ký hiệu & $\begin{array}{c}\text { Số biến } \\
\text { quan sát }\end{array}$ & $\begin{array}{c}\text { Cronbach's } \\
\text { Alpha }\end{array}$ & $\begin{array}{c}\text { Hệ số tương } \\
\text { quan biến - } \\
\text { tống nhỏ nhất }\end{array}$ \\
\hline 1 & Thông tin minh bạch & TT & 4 & 0,850 & 0,596 \\
\hline 2 & Kiến thức về thực phẩm hữu cơ & KT & 7 & 0,835 & 0,538 \\
\hline 3 & Chuẩn chủ quan & CQ & 4 & 0,730 & 0,470 \\
\hline 4 & Thái độ & TD & 7 & 0,861 & 0,396 \\
\hline 5 & Niềm tin & NT & 4 & 0,850 & 0,652 \\
\hline 6 & Ý định mua & YD & 3 & 0,832 & 0,616 \\
\hline
\end{tabular}

Nguồn: Tính toán của nhóm tác giả.

\subsection{Phân tích nhân tố khám phá (EFA)}

Phân tích nhân tố được thực hiện với phép trích Principal Axis Factoring, phép xoay Promax cho 29 biến quan sát. Kết quả phân tích EFA cho thấy các biến TD7, CQ1, CQ2, CQ3, CQ4, TD7, KT4, KT3 bị loại khỏi mô hình do có trọng số không đạt yêu cầu. Phân tích nhân tố cho thấy hệ số $\mathrm{KMO}=0,880$ đạt yêu cầu $(>0,05)$; mức ý nghĩa của kiểm định Bartlett $=0,000(>0,05)$; tổng phương sai trích được là $58,543 \%$; hệ số tải nhân tố đều $>0,5$ nên đạt yêu cầu. Thang đo chính thức sau khi xử lý EFA gồm 21 biến quan sát như trong Bảng 4 (Hà Nam Khánh Giao và Bùi Nhất Vương, 2019).

Bảng 4. Kết quả phân tích EFA

\begin{tabular}{|c|c|c|c|c|c|c|}
\hline \multirow{2}{*}{$\begin{array}{l}\text { Mã } \\
\text { hóa }\end{array}$} & \multirow{2}{*}{ Biến quan sát } & \multicolumn{5}{|c|}{ Yếu tố } \\
\hline & & 1 & 2 & 3 & 4 & 5 \\
\hline $\mathrm{TD}_{3}$ & $\begin{array}{l}\text { Tiêu dùng thực phẩm hữu cơ tốt cho sức khỏe hơn } \\
\text { thực phẩm thông thường. }\end{array}$ & 0,890 & & & & \\
\hline $\mathrm{TD}_{2}$ & $\begin{array}{l}\text { Tiêu dùng thực phẩm hữu cơ an toàn hơn thực phẩm } \\
\text { thông thường. }\end{array}$ & 0,845 & & & & \\
\hline $\mathrm{TD}_{5}$ & $\begin{array}{l}\text { Thực phẩm hữu cơ có chất lượng cao hơn thực phẩm } \\
\text { thông thường. }\end{array}$ & 0,704 & & & & \\
\hline $\mathrm{TD}_{6}$ & $\begin{array}{l}\text { Thực phẩm hữu cơ đắt tiền hơn thực phẩm thông } \\
\text { thường. }\end{array}$ & 0,651 & & & & \\
\hline $\mathrm{TD}_{1}$ & $\begin{array}{l}\text { Thực phẩm hữu cơ có ít dư lượng hóa học hơn thực } \\
\text { phẩm thông thường. }\end{array}$ & 0,585 & & & & \\
\hline $\mathrm{KT}_{5}$ & Tôi biết quá trình sản xuất ra thực phẩm hữu cơ. & & 0,783 & & & \\
\hline $\mathrm{KT}_{6}$ & $\begin{array}{l}\text { Tôi có thể phân biệt thực phẩm hữu cơ so với thực } \\
\text { phẩm thông thường. }\end{array}$ & & 0,752 & & & \\
\hline $\mathrm{KT}_{1}$ & Cá nhân tôi rất am hiểu về thực phẩm hữu cơ. & & 0,692 & & & \\
\hline $\mathrm{KT}_{7}$ & $\begin{array}{l}\text { Tôi có thể nhận diện được bao bì, nhãn mác thực } \\
\text { phẩm hữu cơ. }\end{array}$ & & 0,600 & & & \\
\hline
\end{tabular}


$\mathrm{KT}$ Trung bình người ở thành phố Long Xuyên là rất am hiểu về thực phẩm hữu cơ.

Nhãn mác, bao bì của thực phẩm hữu cơ cung cấp đúng

$\mathrm{TT}_{2}$ thông tin về ngày sản xuất sản phẩm.

Nhãn mác, bao bì của thực phẩm hữu cơ cung cấp đầy

$\mathrm{TT}_{3}$ đủ thông tin về sản phầm.

Nhãn mác, bao bì của thực phẩm hữu cơ cung cấp

$\mathrm{TT}_{1}$ thông tin chính xác về sản phẩm.

Tôi hài lòng với những thông tin trên nhãn mác, bao

$\mathrm{TT}_{4}$ bì của thực phẩm hữu cơ cung cấp. $\mathrm{NT}_{3}$ Tôi tin tưởng chất lượng thực phẩm hữu cơ có bao bì

Tôi tin tưởng các tổ chức chứng nhận thực phẩm

$\mathrm{NT}_{4}$ hữu cơ.

Tôi tin tưởng những người bán thực phẩm hữu cơ có

$\mathrm{NT}_{2}$ chứng nhận chất lượng.

Tôi nghĩ rằng các công ty trong lĩnh vực thực phẩm

$\mathrm{NT}_{1}$ hữu cơ nhận thức được trách nhiệm của họ.

0,594

$\mathrm{YD}_{2}$ Tôi sẵn sàng mua thực phẩm hữu cơ mặc dù giá cao

$\mathrm{YD}_{3}$ Xác suất tôi sẽ mua thực phẩm hữu cơ là rất cao.

Nếu thực phẩm hữu cơ có sẵn tại các siêu thị, cửa hàng thực phẩm tôi sẽ mua chúng.

Nguồn: Tính toán của nhóm tác giả.

\subsection{Kết quả nghiên cứu và thảo luận}

Các thang đo khái niệm nghiên cứu trước tiên được đánh giá bằng hệ số tin cậy Cronbach' alpha và tiếp tục được kiểm định (độ tin cậy tổng hợp, tính đơn nguyên, giá trị hội tụ, và giá trị phân biệt) thông qua phương pháp phân tích nhân tố khẳng định $\mathrm{CFA}$ (Confirmatory Factor Analysis). Phương pháp phân tích mô hình cấu trúc tuyến tính SEM (Structural Equation Modeling) được sử dụng để kiểm định mô hình lý thuyết cùng với các giả thuyết.

Bảng 5. Tóm tắt kết quả kiểm định thang đo các khái niệm trong mô hình

\begin{tabular}{|c|c|c|c|c|c|}
\hline \multirow[b]{2}{*}{ Khái niệm } & \multirow{2}{*}{$\begin{array}{c}\text { Số biến } \\
\text { quan } \\
\text { sát }\end{array}$} & \multicolumn{2}{|c|}{ Độ tin cậy } & \multirow{2}{*}{$\begin{array}{c}\text { Phưong } \\
\text { sai } \\
\text { trích } \\
(\%)\end{array}$} & \multirow[b]{2}{*}{ Giá trị } \\
\hline & & Cronbach & $\begin{array}{c}\text { Tổng } \\
\text { họpp }\end{array}$ & & \\
\hline Thái độ & 5 & 0,864 & 0,868 & 0,57 & \multirow{5}{*}{$\begin{array}{l}\text { Đạt } \\
\text { yêu } \\
\text { cầu }\end{array}$} \\
\hline Kiến thức về thực phẩm hữu cơ & 5 & 0,815 & 0,817 & 0,47 & \\
\hline Thông tin minh bạch & 4 & 0,850 & 0,852 & 0,59 & \\
\hline Niềm tin & 4 & 0,850 & 0,852 & 0,59 & \\
\hline Ý định & 3 & 0,832 & 0,838 & 0,63 & \\
\hline
\end{tabular}


Kết quả CFA cho thấy mô hình đạt độ tương thích với dữ liệu: $\chi^{2[179]}=343,645$; Chi-square/df $=1,920<3$, với giá trị $\mathrm{p}=0,000 ; \mathrm{GFI}=0,881$; $\mathrm{CFI}=0,932 ; \mathrm{RMSEA}=0,062$. Trong đó, các thang đo thông tin minh bạch, kiến thức về thực phẩm hữu cơ, thái độ, niềm tin, ý định mua đều đạt được tính đơn hướng. Hơn nữa, kết quả cho thấy các trọng số $(\lambda i)$ chuẩn hóa đều đạt tiêu chuẩn cho phép $(\geq 0,50)$ và có ý nghĩa thống kê các giá trị p đều bằng 0,000 . Vì vậy, các biến quan sát dùng để đo lường các thang đo đều đạt giá trị hội tụ. Hệ số tương quan giữa các khái niệm đều khác biệt so với 1 ở độ tin cậy 95\%. Vì vậy, các khái niệm thái độ, kiến thức về thực phẩm hữu cơ, thông tin minh bạch, niềm tin và ý định mua đều đạt được giá trị phân biệt (Steenkamp và Trijp, 1991).

Kết quả SEM cho thấy mô hình này đạt độ tương thích với dữ liệu thị trường: $\chi^{2[182]}=$ 370,789 ; Chi-square $/ \mathrm{df}=2,037<3$ với giá trị $\mathrm{p}$ $=0,000 ; \mathrm{GFI}=0,873>0,8 ; \mathrm{CFI}=0,922>0,9$; và $\mathrm{RMSEA}=0,066<0,08$ (xem Hình 2 ). Như vậy, mô hình này phù hợp với dữ liệu thu thập (Hà Nam Khánh Giao và Bùi Nhất Vương, 2019).

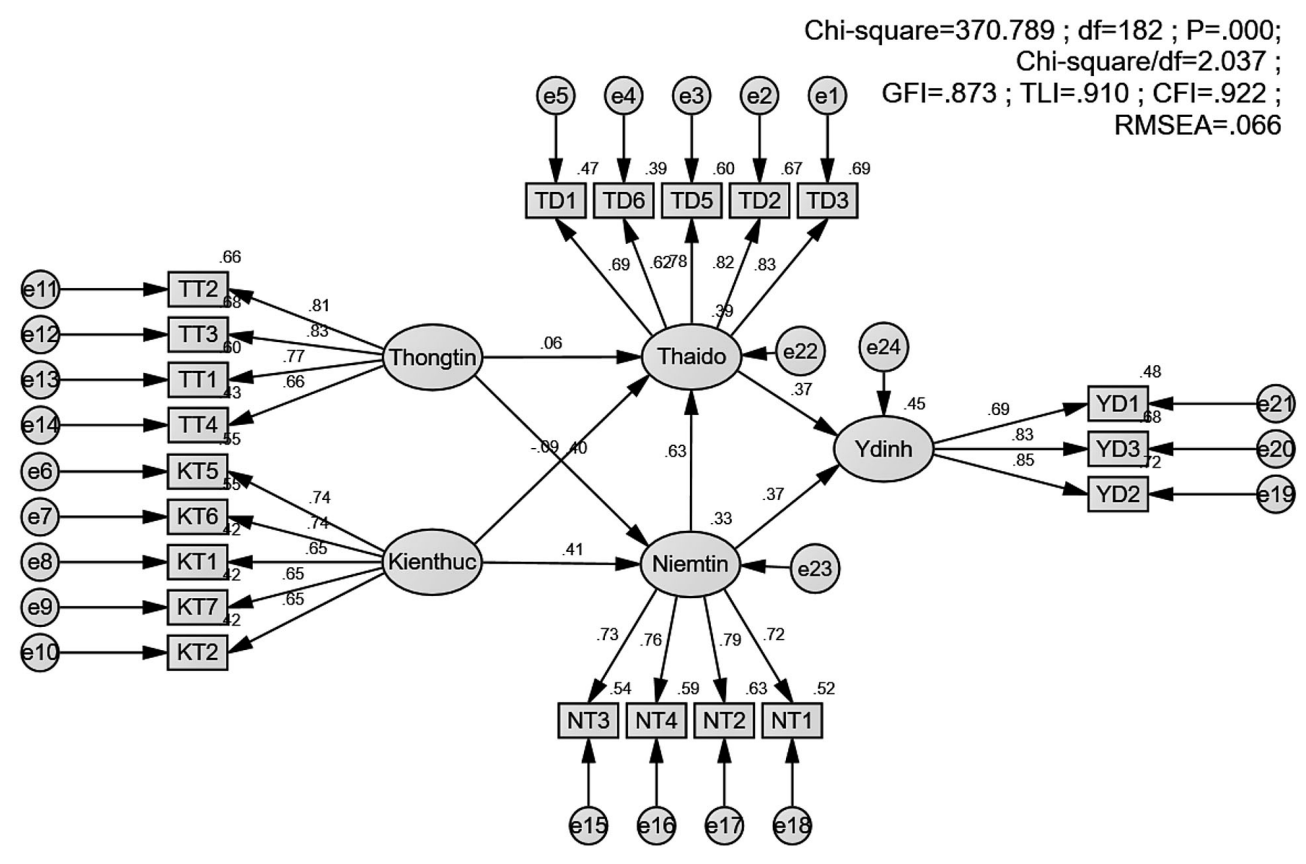

Hình 2. Kết quả kiểm định SEM mô hình nghiên cứu (chuẩn hóa)

Nguồn: Tính toán của nhóm tác giả.

Phân tích cấu trúc tuyến tính (SEM) được sử dụng để kiểm định các giải thuyết nghiên cứu. Kết quả kiểm định cho thấy có năm mối quan hệ giữa các khái niệm đề ra trong mô hình nghiên cứu được chấp nhận ở mức ý nghĩa thống kê $\mathrm{p}=0,000<0,05$ (xem Bảng 6). Kết quả này phù hợp với nghiên cứu của Teng và Wang (2015); Agarwal (2019); Effendi và cs. (2015); Hoàng Thị Bảo Thoa và cs. (2019); Ajzen (1991); Secapramana và Katargo (2019); Nguyễn Kim Nam (2015). Đồng thời, kết quả kiểm định cho thấy thông tin minh bạch và kiến thức về thực phẩm hữu cơ không ảnh hưởng đáng kể đến thái độ. Kết quả nghiên cứu này tương tự kết quả của Teng và Wang (2015). Điều này cho thấy mức độ hiểu biết cao hơn về thực phẩm hữu cơ và những thông tin cung cấp trên nhãn thực phẩm hữu cơ không thể trực tiếp dẫn đến thái độ tích cực hơn đối với thực phẩm hữu cơ. Đối với người tiêu dùng tại thành phố Long Xuyên, khái niệm thực phẩm hữu cơ tương đối mới, kiến thức có thể tạo ra thái độ tích cực chỉ 
khi người tiêu dùng có niềm tin vào thực phẩm với thực phẩm hữu cơ, nếu họ không có niềm hữu cơ. Bên cạnh đó, những thông tin cung cấp tin vào các thông tin ghi trên nhãn thực phẩm trên nhãn thực phẩm hữu cơ không trực tiếp dẫn hữu cơ, ngược lại họ sẽ có xu hướng nghi ngờ đến thái độ tích cực hơn của người tiêu dùng với nguồn thông tin được cung cấp.

Bảng 6. Kết quả kiểm định mối quan hệ nhân quả giữa các khái niệm trong nghiên cứu

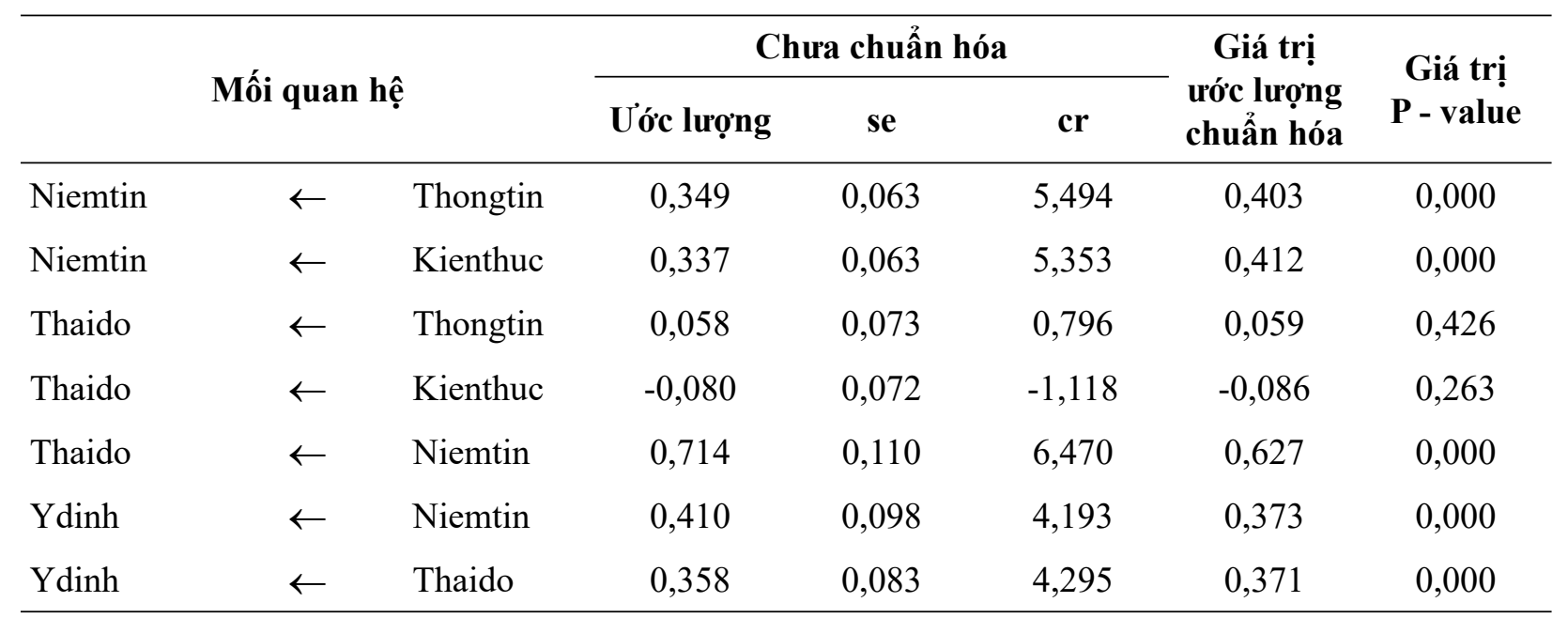

Nguồn: Tính toán của nhóm tác giả.

Kết quả SEM lần 2 cho thấy mô hình này $>0,9$; và RMSEA $=0,066<0,08$ (xem Hình đạt độ tương thích với dữ liệu thu thập: $\chi^{2[184]}$ $=372,574 ;$ Chi-square $/ \mathrm{df}=2,025<3$ với giá
thị $\mathrm{p}=0,000 ; \mathrm{GFI}=0,873>0,8 ; \mathrm{CFI}=0,922 \quad$ Vượng, 2019). 3). Như vậy, mô hình này phù hợp với dữ liệu

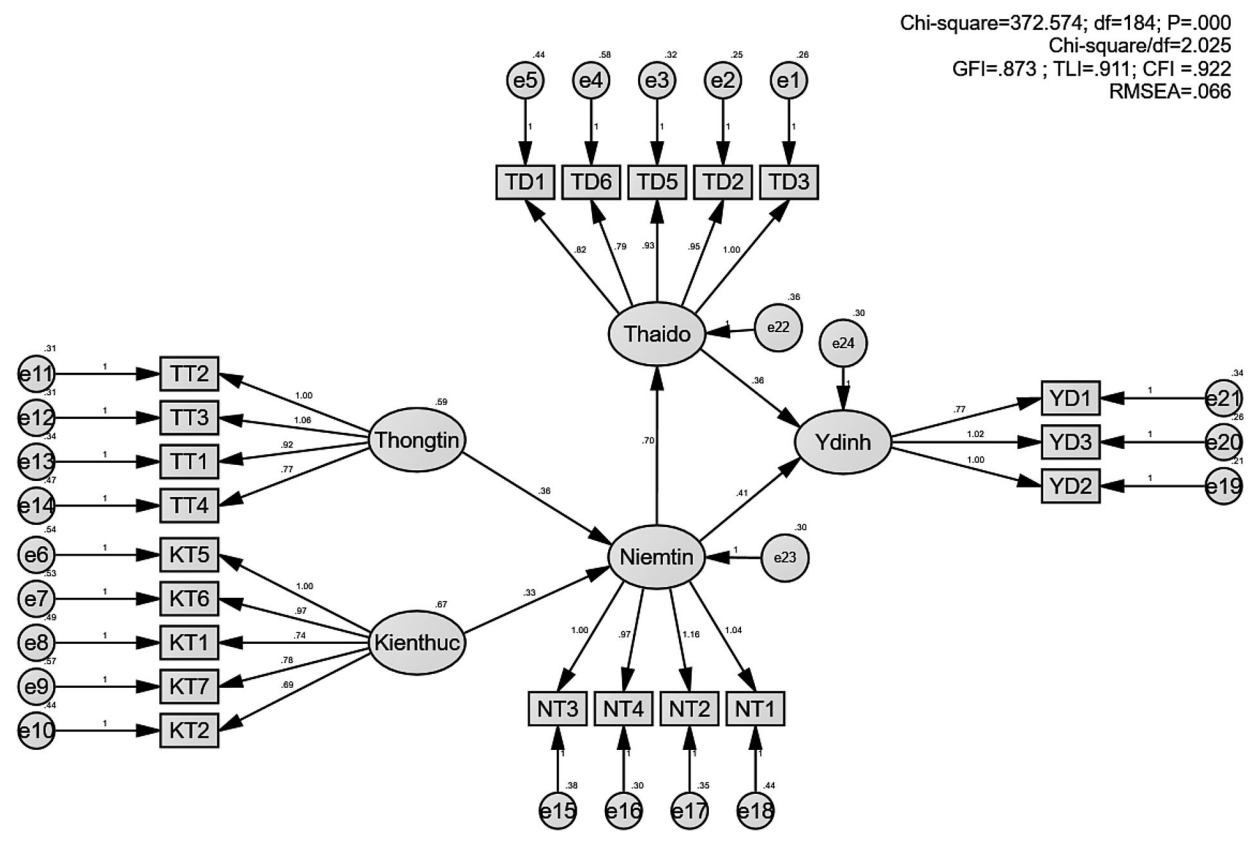

Hình 3. Kết quả kiểm định SEM lần 2 (chuẩn hóa)

Nguồn: Tính toán của nhóm tác giả. 
Bảng 7. Kết quả kiểm định mối quan hệ nhân quả giữa các khái niệm trong nghiên cứu

\begin{tabular}{|c|c|c|c|c|c|c|c|}
\hline & \multirow{2}{*}{ Mối quan hệ } & & \multicolumn{3}{|c|}{ Chưa chuẩn hóa } & \multirow{2}{*}{$\begin{array}{c}\text { Giá trị } \\
\text { ước lượng } \\
\text { chuẩn hóa }\end{array}$} & \multirow{2}{*}{$\begin{array}{c}\text { Giá trị } \\
\text { P - value }\end{array}$} \\
\hline & & & Ước lượng & se & cr & & \\
\hline Niemtin & $\leftarrow$ & Thongtin & 0,356 & 0,063 & 5,621 & 0,412 & 0,000 \\
\hline Niemtin & $\leftarrow$ & Kienthuc & 0,326 & 0,062 & 5,257 & 0,400 & 0,000 \\
\hline Thaido & $\leftarrow$ & Niemtin & 0,699 & 0,089 & 7,824 & 0,614 & 0,000 \\
\hline Ydinh & $\leftarrow$ & Niemtin & 0,408 & 0,098 & 4,178 & 0,371 & 0,000 \\
\hline Ydinh & $\leftarrow$ & Thaido & 0,360 & 0,083 & 4,321 & 0,373 & 0,000 \\
\hline
\end{tabular}

\section{Hàm ý quản trị}

Dựa vào kết quả nghiên cứu, tác giả đề xuất một số giải pháp nhằm thúc đẩy ý định mua thực phẩm hữu cơ của người tiêu dùng tại địa bàn thành phố Long Xuyên như sau:

Một là, những thông tin ghi trên nhãn đầy đủ và đáng tin cậy có ảnh hưởng khá nhiều lên niềm tin của người tiêu dùng vào TPHC. Ở Việt Nam, thực phẩm hữu cơ là một khái niệm tương đối mới so với thực phẩm thông thường, vì vậy những thông tin trên ghi trên nhãn TPHC càng minh bạch, đầy đủ và đáng tin cậy (ví dụ: Cách thức nông nghiệp được trồng, chế biến sản phẩm và xử lý, tỷ lệ thành phần hữu cơ trong sản phẩm, xuất xứ, hạn sử dụng,...) rất quan trọng để gia tăng niềm tin của người tiêu dùng vào TPHC. Ngoài ra, kết quả nghiên cứu cho thấy việc ghi nhãn hữu cơ rõ ràng được coi là một biện pháp hiệu quả để giúp người tiêu dùng có được thông tin hữu ích và đáng tin cậy trong thị trường $\mathrm{TPHC}$, đặc biệt cho những người không có nhiều kiến thức về TPHC, từ đó thúc đẩy ý định mua TPHC. Vì vậy, các doanh nghiệp kinh doanh trong ngành thực phẩm hữu cơ cần có các biện pháp ghi nhãn TPHC một cách rõ ràng, chính xác thông tin và thể hiện sản phẩm là chất lượng để tăng niềm tin cho người tiêu dùng.

Hai là, khi người tiêu dùng nhận thấy họ có hiểu biết tốt về TPHC, quy trình sản xuất TPHC và có thể phân biệt, nhận diện bao bì TPHC so với thực phẩm thông thường cũng như biết được những lợi ích của TPHC mang lại sẽ ảnh hưởng tích cực đến niềm tin về $\mathrm{TPHC}$, từ đó thúc đẩy ý định mua TPHC. Tuy nhiên, kết quả nghiên cứu cho thấy người tiêu dùng không có nhiều kiến thức về TPHC, cũng như quy trình sản xuất TPHC và cách phân biệt, nhận diện bao bì hay logo TPHC. Vì vậy, các doanh nghiệp trong ngành TPHC nên có các chương trình quảng bá, cung cấp các thông tin chính xác và đáng tin cậy về TPHC thông qua các trang mạng xã hội, tivi, tạp chí, trang web... Hơn nữa, cần cung cấp các kiến thức về nhận diện bao bì hay logo của sản phẩm TPHC và các chứng nhận cho sản phẩm hữu cơ. Từ đó giúp người tiêu dùng hiểu biết và có niềm tin hơn về TPHC, góp phần thúc đẩy ý định mua TPHC của người tiêu dùng.

Ba là, kết quả nghiên cứu cho thấy thái độ có mối quan hệ cùng chiều với ý định mua TPHC. Thái độ đại diện cho những gì người tiêu dùng thích và không thích, và quyết định mua sản phẩm thường dựa trên thái độ của người tiêu dùng (Secapramana và Katargo, 2019). Trong nghiên cứu này thái độ phụ thuộc vào lợi ích sức khỏe, an toàn, mức dư lượng hóa học và giá cả của sản phẩm TPHC. Do đó, doanh nghiệp trong thị trường $\mathrm{TPHC}$ cần cung cấp các sản phẩm chất lượng, thành phần hữu cơ đúng như trên nhãn hữu cơ và có các chiến lược giá hợp lý để thu hút người tiêu dùng.

Bốn là, nghiên cứu cho thấy niềm tin có tác động cùng chiều và mạnh mẽ đến thái độ 
của người tiêu dùng. Kiến thức có thể tạo ra thái độ tích cực đối với TPHC chỉ khi niềm tin có thể được xây dựng vững chắc (Teng và Wang, 2015). Kết quả nghiên cứu cho thấy niềm tin của người tiêu dùng phụ thuộc nhiều vào các chứng nhận chất lượng, tổ chức chứng nhận và bao bì/logo của sản phẩm TPHC. Vì vậy, Chính phủ nên có các quy định về việc cấp giấy phép chứng nhận cho sản phẩm hữu cơ và quy định về thiết kế bao bì hay logo TPHC để người tiêu dùng có thể nhận dạng và tin tưởng vào các sản phẩm hữu cơ có chứng nhận là chất lượng. Bên cạnh đó, các doanh nghiệp trong thị trường $\mathrm{TPHC}$ có thể quảng bá hình ảnh, quy trình sản xuất TPHC để tạo ấn tượng tốt đối với người tiêu dùng.

Năm là, kết quả nghiên cứu cho thấy sự sẵn có của các sản phẩm TPHC tại các siêu thị, cửa hàng thực phẩm được người tiêu dùng đánh giá cao nhất trong các biến đo lường ý định mua TPHC. Vì vậy, các doanh nghiệp nên xây dựng và phát triển hệ thống phân phối hiệu quả để giúp người tiêu dùng thuận tiện tiếp cận với các sản phẩm TPHC, điều này góp phần thúc đẩy ý định mua TPHC.

\section{Hạn chế và hướng nghiên cứu tiếp theo}

Kết quả của nghiên cứu này đóng góp những hiểu biết về TPHC và ý định mua TPHC. Tuy đạt được những kết quả cụ thể nêu trên nhưng đề tài nghiên cứu cũng không tránh khỏi những hạn chế nhất định như sau: Đề tài nghiên cứu chỉ kiểm định được một số nhân tố tác động đến ý định mua TPHC, trên thực tế còn có các yếu tố khác tác động tuy nhiên chưa được đề cập trong nghiên cứu. Do thời gian và nguồn lực có hạn nên kích thước mẫu khảo sát có thể chưa đủ lớn và hạn chế về mặt địa lý. Vì vậy, trong tương lai các nghiên cứu tiếp theo có thể ứng dụng mô hình nghiên cứu này vào các khu vực khác và xem xét thêm các yếu tố khác tác động đến ý định mua TPHC. Bên cạnh đó, có thể nghiên cứu sâu hơn về mối quan hệ giữa ý định mua $\mathrm{TPHC}$ và hành vi mua thực tế của người tiêu dùng./.

\section{Tài liệu tham khảo}

Agarwal, P. (2019). Theory of Reasoned Action and Organic Food Buying in India. Srusti Management Review, 7 (2), 28-37.

Ajzen, I. (1989). Attitudes structure and behavior. In Breckler, S.J. and Greenwald, A.G. (Eds), Attitudes Structure and Function, Springer. New York, NY, 241-274.

Ajzen, I. (1991). The Theory of Planned Behavior. Organizational behavior and human decision processes, 50, 179-211.

Ajzen, I. (2002). Perceived Behavioral Control, SelfEfficacy, Locus of Control, and the Theory of Planned Behavior. Journal ofApplied Social Psycholog, 32 (4), 665-683.

Ajzen, I., and Fishbein, M. (1980). Understanding Attitudes and Predicting Social Behavior. Prentice Hall, Englewood Cliffs, NJ.

Ánh Nguyên. (Ngày 13 tháng 11, 2018). Mô hình rau an toàn ở TP. Long Xuyên. Báo An Giang online. Truy cập từ https://baoangiang.com.vn/ mo-hinh-rau-an-toan-o-tp-long-xuyen-a234359. html.

Demeritt, L. (2002). All Things Organic 2002: A Look at the Organic Consumer. The Hartman Group, Bellevue, WA.

Effendi, I., Ginting, P., Lubis, A.N., and Fachruddin, K.A. (2015). Analysis of Consumer Behavior of Organic Food in North Sumatra Province, Indonesia. Journal of Business and Management, 4 (1), 44-58.

Fishbein, M., and Ajzen, I. (1975). Belief, Attitude, Intention, and Behavior: An Introduction to Theory and Research. Reading, MA: Addison-Wesley.

Gefen, D. (2004). What makes an ERP implementation relationship worthwhile: linking trust mechanisms and ERP usefulness. Journal of Management Information Systems, 21 (1), 263-288.

Gracia, A., and Magistris, T.D. (2008). The demand for organic foods in the South of Italy: A discrete choice model. Food Policy, 33, 386-396.

Hà Nam Khánh Giao và Bùi Nhất Vương. (2019). Giáo trình Cao học Phuoong pháp Nghiên cứu Khoa 
họ trong Kinh doanh- Cập nhật SmartPLS. Hà Nội: NXB Tài chính. DOI: 10.31219/osf.io/ hbj3k. ISBN: 978-604-79-2154-6.

Hart, P., and Saunders, C. (1997). Power and trust: critical factors in the adoption and use of electronic data interchange. Organization Science, 8 (1), 23-42.

Hồ Thị Diệp Quỳnh Châu. (2015). Các yếu tố ảnh hưởng đến ý định mua thưc phẩm hũu co của nguoời tiêu dùng tại TP.HCM. Truy cập từ https:// xemtailieu.com/tai-lieu/cac-yeu-to-anh-huongden-y-dinh-mua-thuc-pham-huu-co-cua-nguoitieu-dung-tai-tp-hom-406187.html.

Hoàng Thị Bảo Thoa, Hoàng Lê Kiên, Nguyễn Thu Uyên, và Nguyễn Thị Uyên. (2019). Các nhân tố ảnh hưởng đến ý định tiêu dùng thực phẩm hữu cơ của người tiêu dùng ở Hà Nội. $V N U$ Journal of Science: Economics and Business, 35 (3), 79-90.

Howard, J.A., Shay, R.P., and Green, C.A. (1988). Measuring the effect of marketing information on buying intentions. Journal of Services Marketing, 2 (4), 27-35.

Hughner, R.S., McDonagh, P., Prothero, A., Shultz, C.J., and Stanton, J. (2007). Who are organic food consumers? A complication and review of why people purchase organic food. Journal of Consumer Behaviour, 6 (2-3), 94-110.

Kim, D.J., Ferrin, D.L., and Rao, H.R. (2008). A trust-based consumer decision-making model in electronic commerce: the role of trust, perceived risk, and their antecedents. Decision Support Systems, 44 (2), 544-564.

Kim, H.Y., and Chung, J.E. (2011). Consumer purchase intention for organic personal care products. Journal of Consumer Marketing, 28 (1), 40-47.

Kramer, R.M. (1999). Trust and distrust in organizations: emerging perspectives, enduring questions. Annual Review of Psychology, 50 (1), 569-598.

Lê Thị Thùy Dung. (2017). Các nhân tố tác động đến ý định mua thưc phẩm hũu cơ của nguời tiêu dùng tại thành phố Đà Nã̃ng. Truy cập từ https://123doc.net//document/4826071-cacnhan-to-tac-dong-den-y-dinh-mua-thuc-phamhuu-co-cua-nguoi-tieu-dung-tai-thanh-pho-danang.htm

Nguyễn Kim Nam. (2015). Ý định tiêu dùng thực phẩm hữu cơ của người tiêu dùng trẻ: vai trò của niềm tin. Tap chi Khoa học và Công nghẹ Đại học Đà Nã̃ng, 8 (93), 104-108.

O'Fallon, M.J., Gursoy, D., and Swanger, N. (2007). To buy or not to buy: impact of labelling on purchasing intentions of genetically modified foods. International Journal of Hospitality Management, 26 (1), 117-130.

Secapramana, L.V.H., and Katargo, A.L.G. (2019). Antecedents affecting organic food purchase intentions. The International Journal of Organizational Innovation, 12 (2), 140-150.

Steenkamp, J-BEM., and Van, Trijp HCM. (1991). The use of LISREL in validating marketing constructs. International Journal of Research in Marketing, 8(4), 283-299.

Švecová, J., and Odehnalová, P. (2019). The determinants of consumer behaviour of students from Brno when purchasing organic food. Review of Economic Perspectives - Národohospodárský obzor, 19 (1). 49-64.

Teng, C.C., and Wang, Y.M. (2015). Decisional factors driving organic food consumption. British Food Journal, 117 (3), 1068-1081.

Thu Lưu. (Ngày 16 tháng 12, 2019). Phòng, chống ngộ độc thực phẩm cuối năm. Thời nay. Truy cập từ https://nhandan.com.vn/baothoinay/ baothoinay-xahoi/baothoinay-xahoi-vande/ item/42575602-phong-chong-ngo-doc-thucpham-cuoi-nam.html

Trịnh Thùy Anh. (2014). Các yếu tố ảnh hưởng đến quyết định mua thực phẩm hữu cơ của khách hàng trên địa bàn thành phố Hồ Chí Minh. Khoa hoc thuong mai, 68, 36-42.

Vermeir, I., and Verbeke, W. (2006). Sustainable food consumption: exploring the consumer 'attitude-behavioural intention' gap. Journal of Agricultural and Environmental Ethics, 19 (2), 169-194. 